\title{
An alternate proof of Statman's finite completeness theorem
}

\author{
B. Srivathsan, Igor Walukiewicz \\ LaBRI, Université de Bordeaux, 351 cours de la libération, 33400 Talence, France
}

\begin{abstract}
Statman's finite completeness theorem says that for every pair of non-equivalent terms of simply-typed lambda-calculus there is a model that separates them. A direct method of constructing such a model is provided using a simple induction on the Böhm tree of the term.
\end{abstract}

Keywords: Simply typed lambda calculus, formal semantics, theory of computation

\section{Introduction}

Statman's finite completeness theorem [5, 6] shows that standard models [1] are strong enough to separate terms, up to $\beta \eta$-reductions. It states that given a simply typed lambda term $M$, there exists a finite standard model such that for every term $N$ that is not $\beta \eta$-equivalent to $M$ there is a variable assignment separating the two terms, that is, making their values in the model different. At the time of publication of this work, a crucial corollary of this theorem was that the $\lambda$-definability conjecture implies the higher order matching conjecture [5, 6, 7. However, $\lambda$-definability was shown to be undecidable later by Loader 2].

The first proof of Statman's theorem appeared in [5]. It was explained in more detail in [6] since the previous proof was considered "not accessible to readers not familiar with this subject". The proof proceeds by defining a suitable syntactic equivalence over the lambda terms. The required model is then the quotient of the set of lambda terms by this equivalence.

Salvati 4 . proves that singleton sets, that is sets of the form $\left\{N \mid N={ }_{\beta \eta} M\right\}$ for a fixed $M$ can be characterized by suitable intersection types. In another paper [3], Salvati gives a notion of recognizability of languages of lambda terms based on these intersection types. He shows that this notion coincides with a notion of recognizability defined in terms of finite standard models. This gives an alternate proof to Statman's theorem.

In this paper, we give yet another proof of this theorem. Our proof carries a semantic flavour, constructing the required model for a term $M$ step-by-step, by performing an induction on the Böhm tree of the $\eta$-long $\beta$-normal form of 
$M$. The Böhm trees are the only syntactic tools used. This proof is very direct, especially in comparison to the existing proofs mentioned above. The proof also gives a slightly stronger result: for every term $M$ there is a model and a variable assignment such that if $N$ evaluates to the same value as $M$ then $M={ }_{\beta \eta} N$.

In Section 2, we give the necessary preliminaries. In Section 3, we define the notion of an extended model, and explain the relation between the elements of the initial model and the extended model. Section 4 contains our proof of the finite completeness theorem.

\section{Simply typed $\lambda$-calculus}

The set of types $\mathcal{T}$ is constructed from a unique basic type 0 using a binary operator $\rightarrow$. Thus 0 is the unique basic type, and if $\alpha, \beta$ are types, then $\alpha \rightarrow \beta$ is also a type. The order of a type is defined by: $\operatorname{order}(0)=1$, and $\operatorname{order}(\alpha \rightarrow \beta)=\max (1+\operatorname{order}(\alpha), \operatorname{order}(\beta))$.

The set of simply typed $\lambda$-terms is defined inductively as follows. For each type $\alpha$, there is a countable set of variables $x^{\alpha}, y^{\alpha}, \ldots$ which are also terms of type $\alpha$. If $M$ is a term of type $\beta$ and $x$ is a variable of type $\alpha$, then $\lambda x^{\alpha} . M$ is a term of type $\alpha \rightarrow \beta$. Such a term is called a $\lambda$-abstraction. If $M$ is a term of type $\alpha \rightarrow \beta$ and $N$ is a term of type $\alpha$ then $M N$ is a term of type $\beta$. Terms of this kind are called applications.

A standard finite model $\mathcal{D}$ is a family of finite sets $\left(D_{\alpha}\right)_{\alpha \in \mathcal{T}}$ indexed by the set of types. $\mathcal{D}$ is determined by $D_{0}$ which is a finite set of elements of type 0 . For types $\alpha, \beta$, the set $D_{\alpha \rightarrow \beta}$ is the set of functions from $D_{\alpha}$ to $D_{\beta}$.

A variable assignment is a function assigning to every variable $x^{\alpha}$ an element of $D_{\alpha}$. If $d$ is an element of $D_{\alpha}$ and $x^{\alpha}$ is a variable of type $\alpha, v\left[d / x^{\alpha}\right]$ denotes the variable assignment which assigns $d$ to $x^{\alpha}$ and is identical to $v$ otherwise.

The interpretation of a simply typed $\lambda$-term $M$ in the model $\mathcal{D}$ and variable assignment $v$ is defined inductively:

- $\llbracket x^{\alpha} \rrbracket_{\mathcal{D}}^{v}=v\left(x^{\alpha}\right)$

- $\llbracket M N \rrbracket_{\mathcal{D}}^{v}=\llbracket M \rrbracket_{\mathcal{D}}^{v} \llbracket N \rrbracket_{\mathcal{D}}^{v}$

- $\llbracket \lambda x^{\alpha} \cdot M \rrbracket_{\mathcal{D}}^{v}$ is a function mapping $d \in D_{\alpha}$ to $\llbracket M \rrbracket_{\mathcal{D}}^{v\left[d / x^{\alpha}\right]}$

We recall the two types of reduction over simply typed $\lambda$-terms.

$\beta$-reduction $(\lambda x . M) N \rightarrow_{\beta} M[N / x]$

$\eta$-reduction $(\lambda x . M x) \rightarrow_{\eta} M$, provided $x$ is not free in $M$.

A lambda term in long normal form is of the shape $\lambda \vec{x} . z M_{1} \ldots M_{k}$ where $M_{1}, \ldots, M_{k}$ are in long normal form, $z$ is a variable, the term $z M_{1} \ldots M_{k}$ is of type 0 and the sequence $\lambda \vec{x}$ might be empty.

For a lambda term $M$ in long normal form, its Böhm tree, $B T(M)$ is defined inductively as follows. If $M=\lambda \vec{x} . z M_{1} \ldots M_{k}$, with $z$ being a variable, then the root of $B T(M)$ is labeled $\lambda \vec{x} . z$ and it has $B T\left(M_{1}\right)$ to $B T\left(M_{k}\right)$ as its children. 
$M$ is said to be uniquely determined in a model $\mathcal{D}$ with a variable assignment $v$ if for all lambda terms $N, \llbracket N \rrbracket_{\mathcal{D}}^{v}=\llbracket M \rrbracket_{\mathcal{D}}^{v}$ iff $N={ }_{\beta \eta} M$.

In the following sections, we prove Statman's finite completeness theorem in a slightly stronger form:

Theorem 1 For every $\lambda$-term $M$, there exists a finite model $\mathcal{D}$ and a variable assignment $v$ such that $M$ is uniquely determined in $\mathcal{D}$ and $v$.

To prove this theorem, we consider a lambda term in long normal form. We construct a model in which all its subterms are uniquely determined. We add a fresh element and alter the interpretations to make the lambda term interpret uniquely to this newly added element.

\section{Extended model}

Consider a lambda term $M$ of type 0 . Let $\mathcal{D}$ be a standard finite model and $v$ a variable assignment, so that $\llbracket M \rrbracket_{\mathcal{D}}^{v}=e$, with $e \in D_{0}$. In general, there exist many lambda terms that interpret to $e$. Our objective is to add a new element to $D_{0}$ and make $M$ interpret to this new element. In addition, the other lambda terms of type 0 should interpret as before. This would ensure that $M$ interprets uniquely to this new element. Intuitively, the other lambda terms should not "notice" a difference between $e$ and this new element. We call this new element $e_{\text {clone }}$. Given a model $\mathcal{D}=\left(D_{\alpha}\right)_{\alpha \in \mathcal{T}}$ and an element $e \in D_{0}$, the extended model $\mathcal{D}^{e}=\left(D_{\alpha}^{e}\right)_{\alpha \in \mathcal{T}}$ is the model determined by $D_{0}^{e}=D_{0} \uplus\left\{e_{\text {clone }}\right\}$. As a consequence of adding this extra element, many new higher order functions are generated. Hence we force the $\lambda$-terms to interpret to those functions that behave identically on $e_{c l o n e}$ and on $e$. In the subsequent sections, we study this new extended model and furnish a variable assignment so that $M$ gets uniquely interpreted to $e_{\text {clone }}$.

\subsection{Relating the models}

Consider the function $f \in D_{0 \rightarrow 0}$ shown in Figure 1. The same figure shows some functions in the extended model $\mathcal{D}^{e}$. The function $f_{1}^{\prime}$ acts the same way as $f$ on all the common elements. However, $f_{1}^{\prime}\left(e_{\text {clone }}\right)$ is not equal to $f_{1}^{\prime}(e)$ which is undesirable. Hence we would like to ignore such a function. The function $f_{2}^{\prime}$ on the other hand acts the same way as $f$ on all the common elements and in addition $f_{2}^{\prime}\left(e_{\text {clone }}\right)$ is equal to $f_{2}^{\prime}(e)$. We consider $f_{2}^{\prime}$ as the representative of $f$ in $\mathcal{D}^{e}$. An interesting case is given by $f_{3}^{\prime}$ that instead of mapping the element to $e$ maps it to $e_{\text {clone. }}$. By the intuition that $e_{\text {clone }}$ is equivalent to $e$, we wish to say that $f_{3}^{\prime}$ is equivalent to $f_{2}^{\prime}$.

We define two notions to relate the elements of the extended model $\mathcal{D}^{e}$ to elements of the original model $\mathcal{D}$ :

- an injection function $\operatorname{in}_{\alpha}: D_{\alpha} \rightarrow D_{\alpha}^{e}$ that for every element $f \in D_{\alpha}$ gives its representative $f^{\prime} \in D_{\alpha}^{e}$, 

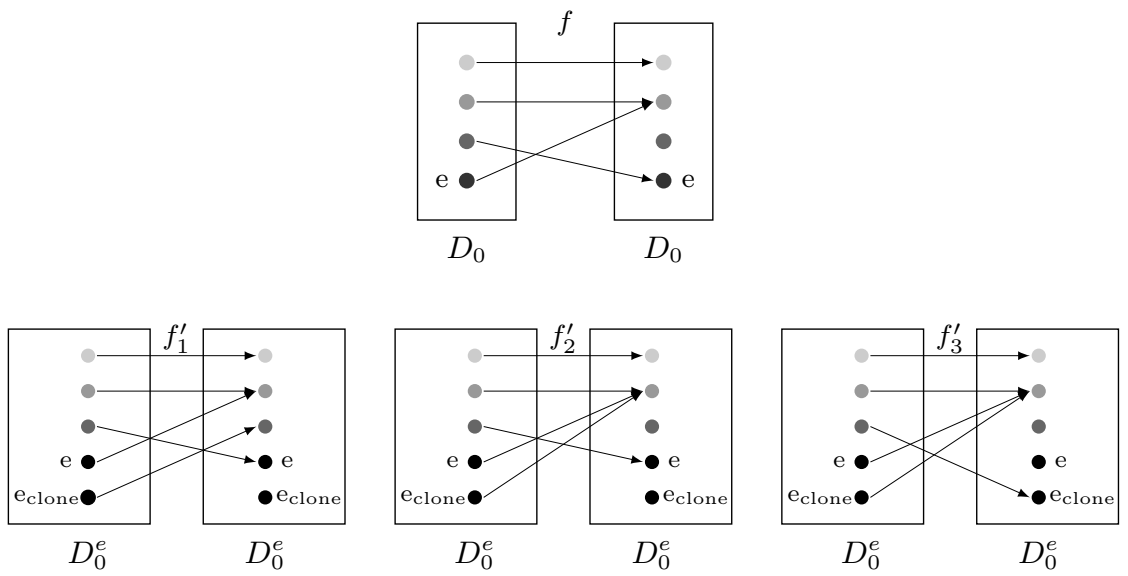

Figure 1: Higher order functions in the extended model $\mathcal{D}^{e}$

- an equivalence relation $\leftrightarrow_{\alpha}$ over $D_{\alpha}^{e}$ that groups $e$ and $e_{\text {clone }}$ at type 0 and propagates this basic equivalence to higher order functions.

In general, we would like to visualize each set $D_{\alpha}^{e}$ as shown in Figure 2 ,

Before formally defining these notions we designate a null element for every type.

Definition 2 The null element $\Delta_{0}$ is any arbitrary element of $D_{0}^{e}$ different from $e_{\text {clone }}$. For a type $\alpha \rightarrow \beta$, element $\Delta_{\alpha \rightarrow \beta}$ is the constant function mapping every element to $\Delta_{\beta}$.

The definitions of in in $_{\alpha}$ and $\leftrightarrow_{\alpha}$ are mutually dependent. For an element $d^{\prime}$ in $D_{\alpha}^{e}$, let $\left[d^{\prime}\right]$ denote the equivalence class of $d^{\prime}$ with respect to $\leftrightarrow_{\alpha}$. For a higher order type $\alpha \rightarrow \beta$ and for a function $f \in D_{\alpha \rightarrow \beta}, \mathbf{i n}_{\alpha \rightarrow \beta}(f)$ maps every element $d^{\prime}$ in $\left[\mathbf{i n}_{\alpha}(d)\right]$ to $\operatorname{in}_{\beta}(f(d))$. We say that a function $f^{\prime} \in D_{\alpha \rightarrow \beta}^{e}$ simulates a function $f \in D_{\alpha \rightarrow \beta}$, written as $\operatorname{sim}\left(f^{\prime}, f\right)$ if $f^{\prime}$ maps every element in an equivalence class $\left[\mathbf{i n}_{\alpha}(d)\right]$ to an element in the equivalence class $\left[\mathbf{i n}_{\beta}(f(d))\right]$. These notions are pictorially represented in Figure 3. The equivalence relation $\leftrightarrow_{\alpha \rightarrow \beta}$ groups functions of $\mathcal{D}^{e}$ that simulate the same function of $\mathcal{D}$. The formal definitions follow.

Definition 3 in $_{\alpha}, \operatorname{sim}_{\alpha}, \leftrightarrow_{\alpha}$

- $\mathrm{in}_{0}, \operatorname{sim}_{0}, \leftrightarrow_{0}$

- in $_{0}: D_{0} \rightarrow D_{0}^{e}$ is the identity.

- $\operatorname{sim}_{0}(d, d)$ for every element $d \in D_{0}$.

- $\leftrightarrow_{0}$ is the smallest equivalence containing $e \leftrightarrow_{0} e_{\text {clone }}$. 


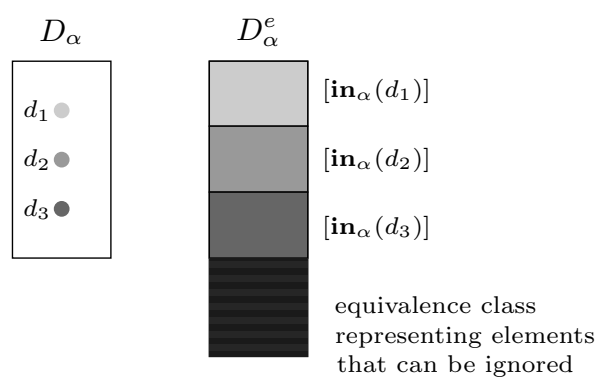

Figure 2: Visualizing a set in the extended model

- $\operatorname{in}_{\alpha \rightarrow \beta}$

For an element $f \in D_{\alpha \rightarrow \beta}, \mathbf{i n}_{\alpha \rightarrow \beta}(f)$ is a function $f^{\prime} \in D_{\alpha \rightarrow \beta}^{e}$ such that for all elements $d^{\prime} \in D_{\alpha}^{e}$,

$$
f^{\prime}\left(d^{\prime}\right)= \begin{cases}\operatorname{in}_{\beta}(f(d)) & \text { if } d^{\prime} \in\left[\operatorname{in}_{\alpha}(d)\right] \\ \Delta_{\beta} & \text { otherwise }\end{cases}
$$

- $\operatorname{sim}_{\alpha \rightarrow \beta}$

For $f \in D_{\alpha \rightarrow \beta}, f^{\prime} \in D_{\alpha \rightarrow \beta}^{e}$, we say $f^{\prime}$ simulates $f$, written as $\operatorname{sim}\left(f^{\prime}, f\right)$, if for all $d \in D_{\alpha}$, for all $d^{\prime} \in\left[\mathbf{i n}_{\alpha}(d)\right]: f^{\prime}\left(d^{\prime}\right) \leftrightarrow_{\beta} \operatorname{in}_{\beta}(f(d))$.

- $\leftrightarrow_{\alpha \rightarrow \beta}$

For $f^{\prime}, g^{\prime} \in D_{\alpha \rightarrow \beta}^{e}, f^{\prime} \leftrightarrow_{\alpha \rightarrow \beta} g^{\prime} \quad$ if for all $h \in D_{\alpha \rightarrow \beta}, \operatorname{sim}\left(f^{\prime}, h\right) \Leftrightarrow \operatorname{sim}\left(g^{\prime}, h\right)$.

Remark 4 Subsequently, we drop the type subscript in in ${ }_{\alpha}, \operatorname{sim}_{\alpha}$ and $\leftrightarrow_{\alpha}$ since it is the same as the type of the elements associated.

Directly from the definitions, we get

Lemma 5 For every $d \in \mathcal{D}$, in $(d)$ simulates $d$.

Before giving a suitable interpretation to the lambda terms in the extended model $\mathcal{D}^{e}$, we need to understand the interplay of the just introduced notions.

Lemma 6 For $d, d_{1}, d_{2} \in D_{\alpha}$ and $d^{\prime} \in D_{\alpha}^{e}$,

1. if $\operatorname{sim}\left(d^{\prime}, d_{1}\right)$ and $\operatorname{sim}\left(d^{\prime}, d_{2}\right)$ then $d_{1}=d_{2}$;

2. $\operatorname{sim}\left(d^{\prime}, d\right)$ if and only if $d^{\prime} \leftrightarrow \operatorname{in}(d)$;

3. If $d_{1} \neq d_{2}$ then $\operatorname{in}\left(d_{1}\right) \leftrightarrow \operatorname{in}\left(d_{1}\right)$.

\section{Proof}

The proof proceeds by induction on the types. The lemma is clear for type 0 . We prove the lemma for a higher order type $\alpha \rightarrow \beta$. Consider $f, f_{1}, f_{2} \in D_{\alpha \rightarrow \beta}$ and $f^{\prime} \in D_{\alpha \rightarrow \beta}^{e}$. 


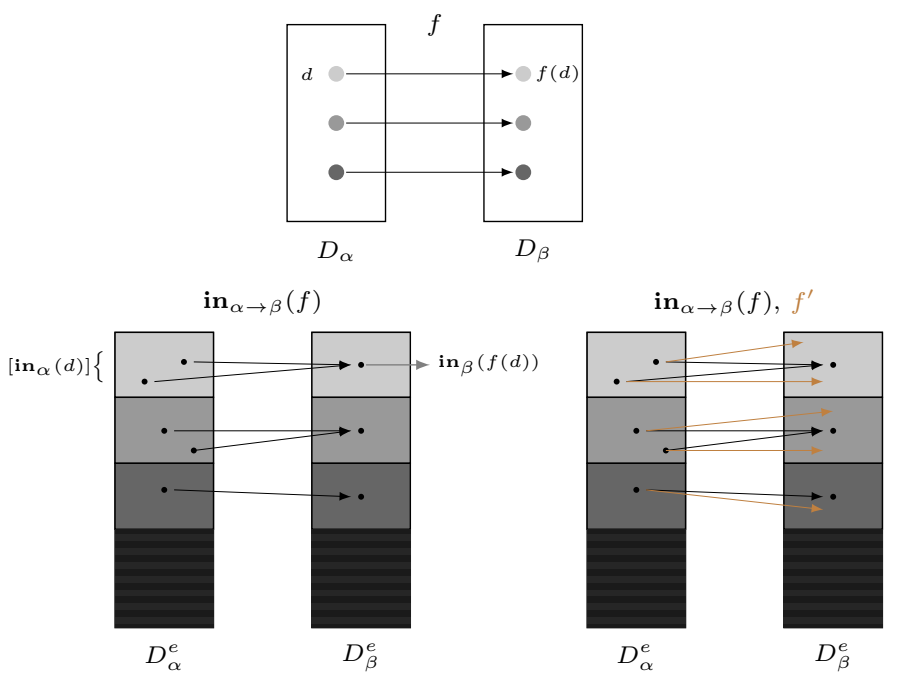

Figure 3: $f, \operatorname{in}_{\alpha \rightarrow \beta}(f), \operatorname{sim}\left(f^{\prime}, f\right)$

1. Suppose $\operatorname{sim}\left(f^{\prime}, f_{1}\right)$ and $\operatorname{sim}\left(f^{\prime}, f_{2}\right)$. Take $d \in D_{\alpha}$ and $d^{\prime} \in[\mathbf{i n}(d)]$. By definition of sim, $f^{\prime}\left(d^{\prime}\right) \leftrightarrow \operatorname{in}\left(f_{1}(d)\right)$ and $f^{\prime}\left(d^{\prime}\right) \leftrightarrow \operatorname{in}\left(f_{2}(d)\right)$. Hence $\operatorname{in}\left(f_{1}(d)\right) \leftrightarrow \operatorname{in}\left(f_{2}(d)\right)$ and by $(3), f_{1}(d)=f_{2}(d)$. Since $d$ is arbitrary, $f_{1}=f_{2}$.

2. For the direction from left to right, suppose $\operatorname{sim}\left(f^{\prime}, f\right)$. By (1) if $\operatorname{sim}\left(f^{\prime}, h\right)$ then $h=f$. Since from Lemma $5, \operatorname{sim}(\mathbf{i n}(f), f)$, the same holds for $\mathbf{i n}(f)$. Therefore, for all $h, \operatorname{sim}\left(f^{\prime}, h\right) \Leftrightarrow \operatorname{sim}(i n(f), h)$ and hence by definition of $\leftrightarrow, f^{\prime} \leftrightarrow \operatorname{in}(f)$.

For the opposite direction, suppose $f^{\prime} \leftrightarrow \mathbf{i n}(f)$. By Lemma 5 , $\operatorname{sim}(\mathbf{i n}(f), f)$ and by definition of $\operatorname{sim}, \operatorname{sim}\left(f^{\prime}, f\right)$.

3. Suppose $f_{1} \neq f_{2}$. From Lemma $5 \operatorname{sim}\left(\mathbf{i n}\left(f_{1}\right), f_{1}\right)$. Hence by (1), not $\operatorname{sim}\left(\operatorname{in}\left(f_{1}\right), f_{2}\right)$. But since $\operatorname{sim}\left(\mathbf{i n}\left(f_{2}\right), f_{2}\right)$, we get $\operatorname{in}\left(f_{1}\right) \leftrightarrow \operatorname{in}\left(f_{2}\right)$.

\subsection{Interpreting the lambda terms in the extended model}

To interpret the lambda terms in $\mathcal{D}^{e}$, we need to define the variable assignment $v^{e}$ that interprets the variables. We intend to pick one from a set of variable assignments that simulate $v$.

Definition 7 A variable assignment $v^{\prime}$ on $\mathcal{D}^{e}$ simulates a variable assignment $v$ on $\mathcal{D}$ if for all variables $x: \operatorname{sim}\left(v^{\prime}(x), v(x)\right)$.

Lemma 8 If $v^{\prime}$ simulates $v$ then for every lambda term $M$ :

$$
\operatorname{sim}\left(\llbracket M \rrbracket_{\mathcal{D}^{e}}^{v^{\prime}}, \llbracket M \rrbracket_{\mathcal{D}}^{v}\right)
$$




\section{Proof}

We proceed by induction on the structure of the lambda term.

1. For variables, the lemma follows from the hypothesis.

2. Consider an application $M N$, with $M$ of type $\alpha \rightarrow \beta$ and $N$ of type $\alpha$. By induction, $\operatorname{sim}\left(\llbracket N \rrbracket_{\mathcal{D}^{e}}^{v^{\prime}}, \llbracket N \rrbracket_{\mathcal{D}}^{v}\right)$ and hence from (2) of Lemma 6 . $\llbracket N \rrbracket_{\mathcal{D}^{e}}^{v^{\prime}} \leftrightarrow \operatorname{in}\left(\llbracket N \rrbracket_{\mathcal{D}}^{v}\right)$. Also by induction, $\operatorname{sim}\left(\llbracket M \rrbracket_{\mathcal{D}^{e}}^{v^{\prime}}, \llbracket M \rrbracket_{\mathcal{D}}^{v}\right)$ and hence from definition, $\llbracket M \rrbracket_{\mathcal{D}^{e}}^{v^{\prime}}\left(\llbracket N \rrbracket_{\mathcal{D}^{e}}^{v^{\prime}}\right) \leftrightarrow \operatorname{in}\left(\llbracket M \rrbracket_{\mathcal{D}}^{v}\left(\llbracket N \rrbracket_{\mathcal{D}}^{v}\right)\right)$. Therefore by (2) of Lemma 6, $\operatorname{sim}\left(\llbracket M N \rrbracket_{\mathcal{D}^{e}}^{v^{\prime}}, \llbracket M N \rrbracket_{\mathcal{D}}^{v}\right)$.

3. Consider a lambda abstraction $\lambda x^{\alpha}$. M. Take $d \in D_{\alpha}$ and $d^{\prime} \in[\operatorname{in}(d)]$. Since $\operatorname{sim}\left(v^{\prime}, v\right)$, we have $\operatorname{sim}\left(v^{\prime}\left[d^{\prime} / x^{\alpha}\right], v\left[d / x^{\alpha}\right]\right)$ and hence by induction $\left.\operatorname{sim}\left(\llbracket M \rrbracket_{\mathcal{D} e^{v^{\prime}}\left[d^{\prime} / x^{\alpha}\right]}\right] \llbracket M \rrbracket_{\mathcal{D}}^{v\left[d / x^{\alpha}\right]}\right)$. From 2) of Lemma 6. $\llbracket M \rrbracket_{\mathcal{D}}^{v^{\prime}\left[d^{\prime} / x^{\alpha}\right]} \leftrightarrow$ $\llbracket M \rrbracket_{\mathcal{D}}^{v\left[d / x^{\alpha}\right]}$. This is true for all $d \in D_{\alpha}$. Hence, by definition, we get $\operatorname{sim}\left(\llbracket \lambda x^{\alpha} \cdot M \rrbracket_{\mathcal{D}^{e}}^{v^{\prime}}, \llbracket \lambda x^{\alpha} \cdot M \rrbracket_{\mathcal{D}}^{v}\right)$

Corollary 9 If $v^{\prime}$ simulates $v$, then every term uniquely determined in $(\mathcal{D}, v)$ is uniquely determined in $\left(\mathcal{D}^{e}, v^{\prime}\right)$.

\section{Proof}

Let $M$ be uniquely determined in $(\mathcal{D}, v)$ but not in $\left(\mathcal{D}^{e}, v^{\prime}\right)$. Therefore, there exists $N \neq_{\beta \eta} M$ such that $\llbracket N \rrbracket_{\mathcal{D}^{e}}^{v^{\prime}}=\llbracket M \rrbracket_{\mathcal{D}^{e}}^{v^{\prime}}$. From Lemma 8 , this would mean that $\operatorname{sim}\left(\llbracket M \rrbracket_{\mathcal{D}^{e}}^{v^{\prime}}, \llbracket M \rrbracket_{\mathcal{D}}^{v}\right)$ and $\operatorname{sim}\left(\llbracket M \rrbracket_{\mathcal{D}^{e}}^{v^{\prime}}, \llbracket N \rrbracket_{\mathcal{D}}^{v}\right)$. Hence by (1) of Lemma 6 . $\llbracket M \rrbracket_{\mathcal{D}}^{v}=\llbracket N \rrbracket_{\mathcal{D}}^{v}$. A contradiction.

\section{Proof of the theorem}

The proof proceeds by an induction on the size of the Böhm tree $B T(M)$ of the lambda term $M$. Let $B T(M)$ contain $m$ nodes. Consider an ordering $s_{1}<\cdots<s_{m}$ of the nodes of $B T(M)$ that is compatible with the child relation: if a node $s_{i}$ is a child of $s_{j}$, then $s_{i}<s_{j}$. Assume that $\mathcal{D}_{k}$ is a model and $v_{k}$ a variable assignment such that all the lambda terms rooted in the nodes $s_{i}$ with $i \leq k$ are uniquely determined in $\left(\mathcal{D}_{k}, v_{k}\right)$. We then construct $\left(\mathcal{D}_{k+1}, v_{k+1}\right)$ where all the lambda terms rooted in the nodes $s_{i}$ with $i \leq k+1$ are uniquely determined. Consequently $M$ gets uniquely determined in $\left(D_{m}, v_{m}\right)$.

\section{Base case}

The base case refers to $\left(\mathcal{D}_{1}, v_{1}\right)$ which uniquely determines a leaf of $B T(M)$. A leaf is variable $z$ of type 0 . Starting with the trivial model $\mathcal{D}_{0}$ which has a singleton $\{\perp\}$ in its basic set and the trivial variable assignment $v_{0}$, we construct the extended model $\mathcal{D}_{0}^{e}$ by adding a new element $\perp_{\text {clone }}$ to the atomic set. The new variable assignment $v_{0}^{e}$ assigns $z$ to $\perp_{\text {clone }}$ and the rest of the variables are maintained with the same interpretation. Clearly, $z$ is uniquely determined in $\left(\mathcal{D}_{0}^{e}, v_{0}^{e}\right)$. Set $\mathcal{D}_{1}$ as $\mathcal{D}_{0}^{e}$ and $v_{1}$ as $v_{0}^{e}$. 
Induction case

Let the lambda term rooted at $s_{k}$ be $\lambda \vec{x} . y M_{1} \ldots M_{n}$ and let $\llbracket y M_{1} \ldots M_{n} \rrbracket_{\mathcal{D}_{k}}^{v_{k}}=$ $e$. For notational simplicity let $\mathcal{D}=\mathcal{D}_{k}$ and $v=v_{k}$. By induction hypothesis, $M_{1}, \ldots, M_{n}$ are uniquely determined in $(\mathcal{D}, v)$.

Construct the extended model $\mathcal{D}^{e}$ by adding an element $e_{\text {clone }}$ to the basic set $D_{0}$ of $\mathcal{D}$. Consider the variable assignment $v^{e}$ defined below.

- $v^{e}(x)=\operatorname{in}(v(x))$, if $x \neq y$.

- For the variable $y$,

$$
v^{e}(y)\left(d_{1}^{\prime}, \ldots, d_{n}^{\prime}\right)= \begin{cases}e_{\text {clone }} & \text { if } d_{i}^{\prime} \in\left[\operatorname{in}\left(\llbracket M_{i} \rrbracket_{\mathcal{D}}^{v}\right)\right], \\ & \text { for } i \in\{1, \ldots, n\} \\ \operatorname{in}(v(y))\left(d_{1}^{\prime}, \ldots, d_{n}^{\prime}\right) & \text { otherwise }\end{cases}
$$

Since $e_{\text {clone }} \leftrightarrow e, v^{e}$ simulates $v$. Hence we infer the following.

1. From Lemma 8, for every lambda term $N, \llbracket N \rrbracket_{\mathcal{D}^{e}}^{v^{e}}$ simulates $\llbracket N \rrbracket_{\mathcal{D}}^{v}$, and hence from Lemma 6

$$
\llbracket N \rrbracket_{\mathcal{D}^{e}}^{v^{e}} \leftrightarrow \operatorname{in}\left(\llbracket N \rrbracket_{\mathcal{D}}^{v}\right)
$$

2. $\llbracket y M_{1} \ldots M_{n} \rrbracket_{\mathcal{D}^{e}}^{v^{e}}=e_{\text {clone }}$.

We now prove that $\llbracket y M_{1} \ldots M_{n} \rrbracket_{\mathcal{D}^{e}}^{v^{e}}$ is uniquely interpreted to $e_{\text {clone }}$. Let $z N_{1} \ldots N_{p}$ be a lambda term such that $\llbracket z N_{1} \ldots N_{p} \rrbracket_{\mathcal{D}^{e}}^{v^{e}}=e_{\text {clone }}$. We first prove that in this case, $z$ has to be equal to $y$.

If $z \neq y$, then $v^{e}(z)=\operatorname{in}(v(z))$. From the definition of the injection function in, $v^{e}(z)\left(x_{1}, \ldots, x_{p}\right)$ is not equal to $e_{\text {clone }}$ whenever each $x_{i}$ simulates some element of $\mathcal{D}$. Recall now that by choice, the null element (c.f. Definition 2 ) $\Delta_{0}$ is not equal to $e_{\text {clone }}$. So even if some $x_{i}$ does not simulate an element of $\mathcal{D}$, $v^{e}(z)\left(x_{1}, \ldots, x_{p}\right)$ which now equals the null element $\Delta_{0}$ cannot be $e_{\text {clone }}$.

Therefore, $z$ equals $y$ and hence $p$ equals $n$. We show that $N_{i}={ }_{\beta \eta} M_{i}$ for all $i$. Now, if $\llbracket N_{i} \rrbracket_{\mathcal{D}_{e}}^{v^{e}} \notin\left[\operatorname{in}\left(\llbracket M_{i} \rrbracket_{\mathcal{D}}^{v}\right)\right]$ for some $i$, by the same reasoning as above, $\llbracket z N_{1} \ldots N_{p} \rrbracket_{\mathcal{D}^{e}}^{v^{e}}$ cannot be equal to $e_{\text {clone }}$. Therefore, $\llbracket N_{i} \rrbracket_{\mathcal{D}_{e}}^{v^{e}} \leftrightarrow \operatorname{in}\left(\llbracket M_{i} \rrbracket_{\mathcal{D}}^{v}\right)$ for all $i$. In addition, from Lemma 8 , we know that $\llbracket N_{i} \rrbracket_{\mathcal{D}_{e}}^{v^{e_{e}}} \leftrightarrow \operatorname{in}\left(\llbracket N_{i} \rrbracket_{\mathcal{D}}^{v}\right)$ too. Hence from the third part of Lemma $6 \llbracket \llbracket N_{i} \rrbracket_{\mathcal{D}}^{v}=\llbracket M_{i} \rrbracket_{\mathcal{D}}^{v}$. From the assumption that each $M_{i}$ is uniquely determined in $(\mathcal{D}, v)$, one can deduce that $N_{i}={ }_{\beta \eta} M_{i}$ for $i \in\{1, \ldots, n\}$. We hence infer that $y M_{1} \ldots M_{k}$ is uniquely determined in $\left(\mathcal{D}^{e}, v^{e}\right)$.

From the fact that $y M_{1} \ldots M_{k}$ is uniquely determined in $\left(\mathcal{D}^{e}, v^{e}\right)$, we can infer that $\lambda \vec{x} \cdot y M_{1} \ldots M_{k}$ is uniquely determined too. To see this, consider a lambda term $\lambda \vec{x} . N$ such that $\llbracket \lambda \vec{x} . N \rrbracket_{\mathcal{D}^{e}}^{v^{e}}=\llbracket \lambda \vec{x} . y M_{1} \ldots M_{k} \rrbracket_{\mathcal{D}^{e}}^{v^{e}}$. This means that both $N$ and $y M_{1} \ldots M_{k}$ with $\vec{x}$ substituted by values from $v^{e}$ interpret to the same element of $\mathcal{D}^{e}$. That is, $\llbracket N \rrbracket_{\mathcal{D}^{e}}^{v^{e}}=\llbracket y M_{1} \ldots M_{k} \rrbracket_{\mathcal{D}^{e}}^{v^{e}}$. Hence, $N={ }_{\beta \eta}$ $y M_{1} \ldots M_{k}$ as the latter term is uniquely determined in $\left(\mathcal{D}^{e}, v^{e}\right)$.

Set $\mathcal{D}_{k+1}=\mathcal{D}^{e}$ and $v_{k+1}=v^{e}$. Therefore, from the above argument and from Corollary 9, the lambda terms rooted at nodes $s_{i}$ with $i \leq k+1$ are uniquely determined in $\left(\mathcal{D}_{k+1}, v_{k+1}\right)$, thus proving the inductive step. 


\section{References}

[1] L. Henkin. Completeness in the theory of types. Journal of symbolic logic, 15(2):81-91, 1950.

[2] R. Loader. The undecidability of-definability. Logic, meaning, and computation: essays in memory of Alonzo Church, page 331, 2001.

[3] S. Salvati. Recognizability in the simply typed lambda-calculus. Logic, Language, Information and Computation, pages 48-60, 2009.

[4] S. Salvati. On the membership problem for non-linear Abstract Categorial Grammars. Journal of Logic, Language and Information, 19(2):163-183, 2010.

[5] R. Statman. Completeness, invariance and $\lambda$-definability. Journal of Symbolic Logic, 47(1):17-26, 1982.

[6] R. Statman and G. Dowek. On Statman's Finite Completeness Theorem. Carnegie Mellon University, School of Computer Science, 1992.

[7] DA Wolfram. The clausal theory of types. PhD thesis, 1989. 